\title{
Feira de projetos de intervenção: a experiência do ensino sobre formulação, gestão e avaliação de projetos públicos
}

\author{
Mauro César da Silveira1 \\ Marcos Arcanjo de Assis ${ }^{2}$ \\ Fundação João Pinheiro
}

\begin{abstract}
RESUMO
O artigo discute um caso de ensino sobre o processo de formação de políticas públicas em um curso de Administração Pública, sob a perspectiva da interdisciplinaridade. Trata-se da Feira de Projetos de Intervenção, trabalho desenvolvido no âmbito de duas disciplinas em que os alunos selecionam um problema público relevante e, aplicando metodologias de concepção e gerenciamento de projetos, desenham uma alternativa de solução. O objetivo do texto é registrar a experiência e, a partir dela, discutir aspectos relevantes para o desenvolvimento de estratégias interdisciplinares de ensino em cursos do Campo de Públicas. Adota-se uma perspectiva de interdisciplinaridade que vai além da interação entre distintas disciplinas, sendo fundamental, a troca, reciprocidade, diálogo e disposição para inovação dos envolvidos. Duas metodologias são aplicadas no desenvolvimento do projeto da Feira: o Marco Lógico, que fornece referências objetivas para a concepção, gestão e avaliação de projetos; e as boas práticas do Project Management Institute, método baseado em áreas de conhecimentos e grupos de processos adequados ao gerenciamento de projetos. Cinco edições dessa experiência já foram realizadas, resultando no desenho de 89 projetos. A cada edição, inovações na dinâmica foram incorporadas, ampliando as potencialidades e os aprendizados sobre a interdisciplinaridade na Administração Pública. Destacam-se a importância da articulação de teorias e métodos para compreensão de situações-problema complexas, a experiência, mesmo simulada, dos reveses da prática administrativa no setor público, a relevância da abertura para a inovação e para o trabalho em parceria entre os docentes envolvidos, além de dificuldades instrumentais sobre o ensino do processo de produção de políticas públicas.
\end{abstract}

Palavras-chave: Caso de ensino. Administração Pública. Gestão e avaliação de projetos. Marco Lógico. PMI.

\begin{abstract}
The article discusses a teaching case on the process of public policy formation in a Public Administration course, from the perspective of interdisciplinarity. This is the Intervention Projects Fair, work developed within the framework of two disciplines in which students select a relevant public problem and, applying design methodologies and project management, design an alternative solution. The aim of the text is to record the experience and, based on it, discuss aspects relevant to the development of interdisciplinary teaching strategies in courses in the Public Field. An interdisciplinary perspective is adopted that goes beyond the interaction between different disciplines, being fundamental, the exchange, reciprocity, dialogue and willingness to innovation of those involved. Two methodologies are applied in the development of the Fair project: The Logical Framework, which provides objective references for the conception, management and evaluation of projects; and the good practices of the Project Management Institute, a method based on areas of knowledge and process groups suitable for project management. Five editions of this experience have already been carried out, resulting in the design of 89 projects. In each edition, innovations in dynamics have been incorporated, expanding the potential and learning about interdisciplinarity in Public Administration. The importance of articulating theories and methods for understanding complex problem situations, the experience, even simulated, of the setbacks of administrative practice in the public sector, the relevance of openness to innovation and to work in partnership between the teachers involved, as well as instrumental difficulties on teaching the process of production of public policies, stand out.
\end{abstract}

Keywords: Teaching case. Public Administration. Project management and evaluation. Logical Framework. PMI 


\section{RESUMEN}

El artículo analiza un caso de enseñanza sobre el proceso de formación de políticas públicas en un curso de Administración Pública, desde la perspectiva de la interdisciplinariedad. Es la Feria de Proyectos de Intervención, trabajo desarrollado en dos disciplinas en las que los estudiantes seleccionan un problema público relevante y, aplicando el diseño del proyecto y las metodologías de gestión, diseñan una solución alternativa. El objetivo del texto es registrar la experiencia y, a partir de ella, discutir aspectos relevantes para el desarrollo de estrategias de enseñanza interdisciplinar en los cursos de campo público. Adopta una perspectiva de interdisciplinariedad que va más allá de la interacción entre diferentes disciplinas, siendo fundamental el intercambio, la reciprocidad, el diálogo y la voluntad de innovación de los involucrados. Se aplican dos metodologías al proyecto de desarrollo de la Feria: el Marco Lógico, que proporciona referencias objetivas para el diseño, gestión y evaluación de proyectos; y las mejores prácticas del Project Management Institute, un método basado en el conocimiento y un grupo de procesos adecuados para la gestión de proyectos. Ya se han realizado cinco ediciones de este experimento, resultando en el diseño de 89 proyectos. En cada edición, se incorporaron innovaciones en dinámica, ampliando las potencialidades y aprendiendo sobre la interdisciplinariedad en la Administración Pública. Se destaca la importancia de articular teorías y métodos para comprender situaciones problemáticas complejas, la experiencia, incluso simulada, de los contratiempos de la práctica administrativa en el sector público, la relevancia de la apertura a la innovación y el trabajo de colaboración entre los docentes involucrados, así como dificultades instrumentales en la enseñanza del proceso de elaboración de políticas públicas.

Palavras-chave: Caso docente. Administración pública. Gestión y evaluación de proyectos. Marco lógico. PMI

\section{Introdução}

O amplo campo de ensino em Administração Pública é por constituição interdisciplinar, o que se impõe como um dos desafios aos professores que se dedicam à formação de profissionais na área (Pires, et al., 2014). Se por um lado já se chegou ao consenso sobre a necessidade de se conhecer certa dose de economia, teoria política, direito administrativo e administração para compreender o funcionamento da máquina pública e geri-la de maneira eficiente, por outro, articular esses conhecimentos, por vezes abstratos, e coloca-los em ação já não parece ser uma tarefa tão consensual assim. Assim sendo, o desafio dos cursos de administração pública é (re)combinar - construtivamente - esses âmbitos do saber, caminhando para com processos mais interdisciplinares.

Segundo Oliveira e Moreira (2017), outros autores brasileiros têm pesquisado o tema da interdisciplinaridade, mas ainda são poucos os trabalhos no sentido de mostrar sua prática no âmbito da educação superior, sendo este debate mais frequente entre os docentes que atuam na educação básica.

Este artigo relata e discute a experiência de ensino em formulação, gestão e avaliação de projetos públicos realizada com alunos de um curso superior de Administração Pública fundamentado na interdisciplinaridade. O caso consiste na Feira de Projetos de Intervenção, um trabalho interdisciplinar de duas disciplinas obrigatórias do currículo, nas áreas de formulação, gestão e avaliação de políticas em que os alunos aplicam as metodologias aprendidas no desenho de um projeto de intervenção governamental e na estruturação de seu plano de gerenciamento. Os objetivos do texto são, além de relatar e discutir o caso de ensino, analisar as potencialidades e desafios da interdisciplinaridade no contexto dos cursos do Campo de Públicas.

O artigo se divide em três seções. De início, reflete-se sobre a perspectiva de interdisciplinaridade no Campo de Públicas. Na sequência, descreve-se sinteticamente as metodologias trabalhadas com os estudantes para a realização da Feira de Projetos. Em seguida, os objetivos, a dinâmica de funcionamento e as inovações adotadas na experiência são relatados, para, nas considerações finais do texto, discutir-se os aprendizados potenciais da Feira de Projetos para o ensino de Administração Pública a partir da interdisciplinaridade.

\section{A interdisciplinaridade e o ensino de Administração Pública}

De acordo com Fazenda (2003), as primeiras discussões sobre a interdisciplinaridade surgiram na Europa, especialmente na França e na Itália, no século XX, em meados da 
década de 1960, num período assinalado pelos movimentos estudantis que, dentre outras reivindicações, exigiam um ensino mais sintonizado com as questões de ordem social, política e econômica da época. Os estudantes, ao lutarem por uma outra universidade, mais participativa e mais presente na sociedade, conseguiram mobilizar a academia no sentido de buscar respostas pela interdisciplinaridade para os grandes problemas da época que não se resolviam através uma única disciplina ou área do saber. No final da década de 1960, a interdisciplinaridade chegou ao Brasil e logo exerceu influência na elaboração da Lei de Diretrizes e Bases da Educação Nacional - LDB N 5.692/71. De lá para cá, o tema é pauta de estudos sobre inovação educacional quase sempre como um desafio constante para um ensino mais inovador e preocupado com a complexidade da sociedade contemporânea.

Em relação ao Campo de Públicas, a aprovação das Diretrizes Curriculares Nacionais (DCN's) específicas para os cursos deste Campo em dezembro de 2010 impôs a docentes, discentes e pesquisadores o desafio de consolidar uma comunidade científica propriamente dita, multi/interdisciplinar e pluralista. Neste sentido, requer-se dos cursos deste Campo contribuições de diversas áreas de conhecimento e a construção de um fio condutor ou construto teórico que integre as disciplinas (Oliveira, Sant'ana \& Diniz, 2011). Caso este diálogo não se efetive, o curso de administração pública corre o risco de ser apenas uma justaposição de disciplinas que muito pouco contribui para oferecer uma visão harmônica e integrada do campo (Coelho, 2008).

No Brasil, os precursores da interdisciplinaridade são Hilton Japiassu e Ivani Fazenda, ambos com forte influência de Georges Gusdorf (Silva, 2000). Japiassu (1976) define interdisciplinaridade como uma axiomática comum a um grupo de disciplinas conexas e definidas no nível hierárquico imediatamente superior, o que introduz noção de finalidade. Para ele, interdisciplinaridade é um método capaz de fazer com que duas disciplinas interajam entre si a partir da simples comunicação das ideias até a integração de conceitos, epistemologia, terminologia, metodologia, procedimentos, dados e organização da pesquisa. Para Fazenda (2003) é uma atitude de reciprocidade que impele à troca, ao diálogo; atitude de humildade diante da limitação do próprio saber; atitude de perplexidade ante a possibilidade de desvendar novos saberes. Para a autora, o trabalho interdisciplinar repousaria em uma busca constante que, a princípio, requereria que cada professor conhecesse a sua disciplina, para depois conhecer a disciplina de outro e, assim, o trabalho interdisciplinar seria construído.

Neste sentido, a adoção de práticas interdisciplinares no Campo de Públicas visa privilegiar construções com múltiplas disciplinas inter-relacionadas, propiciando debates entre professores, alunos, conteúdos, metodologias e concepções de mundo por meio da articulação em torno de atividades, promovendo interação de saberes e repertórios diferentes.

\section{Conhecendo as disciplinas envolvidas no caso de ensino}

Nos últimos 20 anos, a Administração Pública brasileira passou por várias mudanças institucionais em decorrência da reforma gerencial do estado iniciada em meados dos anos 1990. O aperfeiçoamento dos processos de planejamento e controle governamental, a crescente preocupação com a eficiência e qualidade do gasto público, a profissionalização dos servidores e a intensificação da atuação do estado na provisão de políticas públicas, preconizada pela Constituição de 1998 são alguns dos exemplos das mudanças. Neste contexto, metodologias de formulação, gestão e avaliação de políticas públicas ganham relevância nos currículos dos cursos de formação da área, embora as experiências concretas dos setores governamentais na adoção dessas estratégias permaneçam modestas (Jannuzzi, 2005; 2011).

O Projeto Pedagógico do curso de Administração Pública estudado possui entre os seus componentes curriculares as disciplinas Gestão de Projetos (GP) e Avaliação Social de Projetos (ASP). O projeto pedagógico atual está em vigor desde 2013, sendo que no primeiro ano as disciplinas foram tratadas de forma isolada por cada professor. Já em 2014 , em uma breve reunião, os professores conversaram sobre as ementas e o que deveria ser abordado em cada disciplina para evitar a redundância. Deste então, em GP, trabalha-se com a metodologia de Gerenciamento de Projetos a partir da adaptação de boas práticas em gestão de projetos do PMI (Project Management Institute). Já em ASP, além dos conceitos, 
tipologias, desafios para a institucionalização das ferramentas de monitoramento e avaliação de políticas públicas, apresenta-se, como recurso metodológico de apropriação dos conceitos, a Metodologia do Marco Lógico.

A metodologia do Marco Lógico (MML) é uma ferramenta aplicada à concepção de projetos de intervenção e que provê uma referência objetiva para a gestão, o monitoramento e a avaliação de tais iniciativas. Surgiu no contexto de intensificação dos investimentos em projetos de desenvolvimento de cooperação internacional na América Latina, datados nas décadas de 1960 e 1970, como resposta a três dificuldades recorrentes aos projetos: planejamento imprecisos, com objetivos distintos e desconectados das atividades; implementação fracassada, com pouca clareza sobre as responsabilidades dos gerentes; avaliações com resultados ambíguos, visto que não havia elementos de comparação entre o previsto e o executado.

A MML se fundamenta em uma perspectiva racional do processo de formulação de políticas, isto é, parte da seleção de um problema público e da análise de relações de causa e efeito atribuídas a ele para se definir o tipo mais viável de intervenção a se realizar. Adota uma gramática uniforme, incentivando o processo participativo de desenho e planejamento, facilitando a comunicação e o acompanhamento dos envolvidos. Apesar de uma maior contribuição residir no desenvolvimento da "ideia mais viável" para a solução de um problema, também pode ser utilizada nas outras etapas do ciclo de projeto, sendo fundamental, inclusive, para organizar a execução e fornecer insumos para o monitoramento e a avaliação (Ortegon, Pacheco \& Prieto, 2005).

Para a metodologia, projetos são empreendimentos racionais que intermediam intenções e consequências, isto é, uma situação atual problemática e uma situação futura desejada, onde ocorrem menos problemas. Um projeto tem a capacidade de intervir no contexto atual na medida em que mobiliza um conjunto de insumos, alocados na realização de atividades produtoras de bens, serviços e atividades que visam atacar os fundamentos do problema que caracteriza esse contexto, gerando efeitos e impactos, ou seja, a transformação esperada (Mokate, 2002; Ortegon, Pacheco \& Prieto, 2005). A figura abaixo representa graficamente essa concepção de projetos, denominada por Mokate (2002) como marco conceitual ou cadeia de objetivos.

Figura 1 - A lógica do marco conceitual de projetos

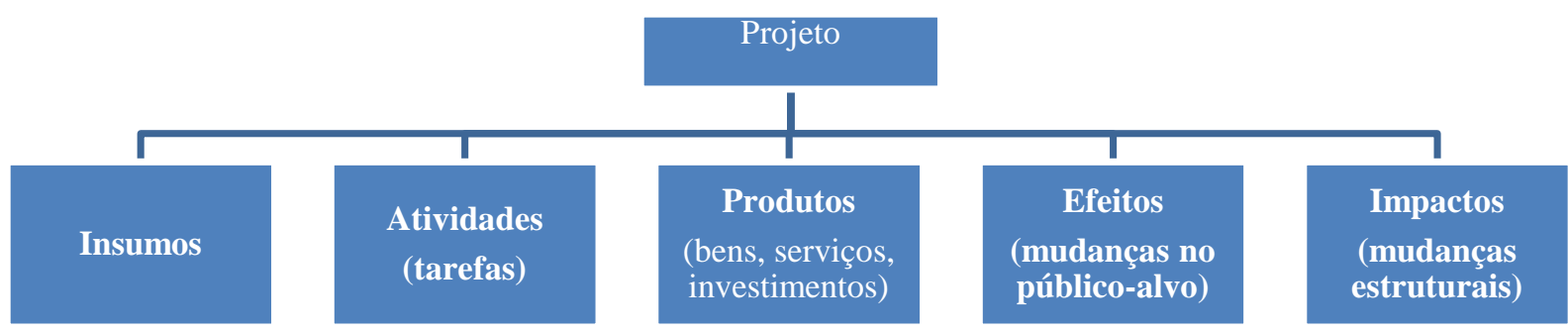

Fonte: Elaborada pelos autores com base em Mokate (2002).

Considerando essa lógica, a MML se organiza em duas etapas e cinco passos. Na primeira etapa, o problema motivador do projeto é identificado e estudado, e a partir do diagnóstico da situação existente, vislumbra-se a situação desejada, para a qual especificamse alternativas de solução. Os passos para a concretização dessa etapa são: análise de envolvidos e análise de problemas (imagem da realidade), análise de objetivos (imagem do futuro) e análise de alternativas (comparação entre diferentes possibilidades de intervenção) (Ortegon, Pacheco \& Prieto, 2005).

A segunda etapa consiste no planejamento da alternativa mais viável em um plano operativo para a implementação. Nela, a matriz do marco lógico é elaborada, onde se especificam os objetivos do projeto, os produtos e atividades, os indicadores de acompanhamento e de resultados e os riscos que podem afetar o seu desenvolvimento (Pfeiffer, 2000). A combinação entre o marco conceitual e a MML pode ser visualizada na figura 2 abaixo: 
Figura 2 - A relação entre o marco conceitual de projetos e a MML

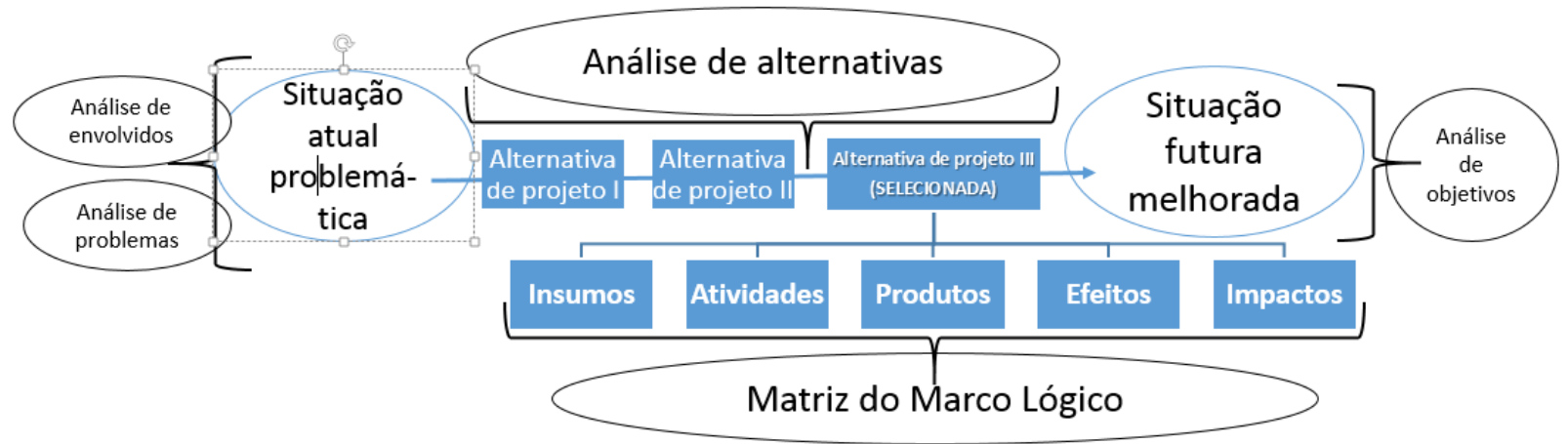

Fonte: Elaborado pelo autor.

Já a metodologia de boas práticas em gerenciamento de projetos do PMI se estrutura a partir da relação entre (i) as diferentes etapas do ciclo de projetos (iniciação, concepção, planejamento, implementação, controle e finalização), (ii) os processos de gerenciamento agrupados a partir da lógica de cada fase e (iii) instrumentos de gerenciamento (Pfeiffer, 2005). De maneira integrada a tais processos, a metodologia propõe distintas áreas de conhecimento que fundamentam a construção e execução de um projeto, isto é, aos elementos essenciais que devem organizar a proposta. Neste sentido, os grupos de processos se sobrepõem e interagem entre si e com as áreas de conhecimento, o que pretende garantir a natureza integradora do gerenciamento de projetos.

A área de gerenciamento de escopo define todo o esforço necessário para entregar os produtos que o projeto propõe, ou seja, "o trabalho que precisa ser realizado pelo projeto" (Pfeiffer, 2005, p. 79). Nela, a razão ou justificativa, os objetivos, a estratégia global (entregas, atividades, metas gerais, prazos) e as partes envolvidas são especificadas. Aqui reside um ponto de confluência entre as duas metodologias empregadas no caso em relato: o quadro lógico fornece os insumos necessários para os processos de planejamento de escopo, uma vez que sintetiza a lógica de intervenção desde os objetivos até a especificação de produtos e atividades.

O gerenciamento de tempo se organiza a partir dos processos relativos à definição e acompanhamento do período de duração do projeto, a partir da estimativa temporal das atividades definidas no escopo, da avaliação das relações de precedência e dependência entre elas e do controle o tempo real gasto na sua execução (Pfeiffer, 2005).

O gerenciamento de custos considera os processos que resguardam que a implementação do projeto ocorra dentro do valor monetário aprovado, tais como: especificação dos recursos necessários para a realização das atividades, estimativas dos custos de tais insumos, elaboração do orçamento e controle dos gastos (Pfeiffer, 2005).

A área de recursos humanos inclui as atividades de organização e gerenciamento da equipe que trabalha projeto, desde a definição das funções e responsabilidades atribuídas a cada uma delas, passando pela seleção e contratação, desenvolvimento, formação e acompanhamento do trabalho dos profissionais (Pfeiffer, 2005).

No gerenciamento de aquisições, deve-se planejar e realizar as compras ou contratações dos insumos necessários para a produção dos bens e serviços definidos no escopo do projeto, levando em conta os prazos do cronograma e os valores estimados no orçamento (Pfeiffer, 2005).

A área de qualidade na gestão de projetos também apresenta confluência com a MML, visto que consiste nos processos de identificação, garantia e controle dos padrões de qualidade da iniciativa, informações que a coluna de indicadores de acompanhamento do quadro lógico fornece ao gerente (Pfeiffer, 2005).

O gerenciamento de riscos consiste nos processos de previsão, análise quantitativa e qualitativa e especificação de contramedidas de situações potenciais que afetam o 
desenvolvimento do projeto, denominadas de riscos. Considera as possibilidades de contornalos caso ocorram, de modo a evitar os seus efeitos. Este é mais um ponto de confluência entre as duas metodologias aplicadas à Feira: a análise de envolvidos da MML serve de insumo para planejamento e controle de riscos, já que os envolvidos podem gerar conflitos no âmbito do projeto que levem a riscos para a consecução dos objetivos e/ou para o desenvolvimento dos produtos (Pfeiffer, 2005).

Os pontos de confluência são elementos que avigoram as possibilidades de se realizar práticas interdisciplinares entre as disciplinas envolvidas no caso de ensino aqui relatado. Os dois professores, além de conhecer o que é trabalhado nas aulas um do outro, se esforçam em fazer as ligações entre os conteúdos, seja mencionando textos da bibliografia de referência, seja ilustrando a aplicação das ferramentas a partir de exemplos comuns. Para que a Feira de Projetos seja realmente interdisciplinar não basta haver uma boa dose de interação entre as disciplinas: é indispensável o diálogo e cooperação, o desejo de inovar, de criar, de ir além de ensinar o que está previsto nas ementas. Acredita-se que esse elemento é um diferencial para o sucesso do caso aqui relatado.

Ainda fazem parte da metodologia de boas práticas do PMI, o gerenciamento de comunicações e partes interessadas. A primeira considera os processos de levantamento e disponibilização das informações do projeto a todos os atores envolvidos, de maneira oportuna e tempestiva. A segunda inclui a identificação de pessoas ou grupos interessados com a temática do projeto, de seus interesses e capacidade de intervenção, de modo a acompanhar o seu envolvimento durante a execução e garantir o seu engajamento (Pfeiffer, 2005).

As duas metodologias aqui expostas já são, há bastante tempo, ensinadas nos cursos de elaboração, gestão e avaliação de projetos. Embora não sejam inéditas no campo, são raras as experiências de aplicação ao setor público e mais ainda, de utilização integrada de suas ferramentas em um mesmo caso prático, como propõe a Feira de Projetos. O comum é propor que os aprendizes concebam o projeto com a MML, sem detalhar o plano de gerenciamento ou que partam de um escopo já especificado para aplicar os processos das boas práticas do PMI, sem experimentar a complexidade da concepção lógica de um projeto do problema motivador à alternativa mais viável. Portanto, a experiência se revela com significativo potencial para que os estudantes de Administração Pública exercitem de maneira mais completa e abrangente o processo de produção de políticas e projetos públicos.

\section{Conhecendo o caminho da interdisciplinaridade no caso}

Como visto na seção anterior, ainda que possa ser evidente a potencialidade do conteúdo das disciplinas serem tratados de maneira interdisciplinar, apenas no segundo semestre de 2014 (três semestres após a adoção do novo projeto pedagógico), foi feita a primeira tentativa do desenvolvimento de um trabalho em conjunto. Nesta experiência, ainda muito tímida, havia um trabalho compartilhado pelas duas disciplinas, ou seja, foi proposto para os alunos um trabalho comum dividido em duas partes, de modo que neste desenho inicial a troca de experiências e a integração dos conteúdos ficavam quase que exclusivamente por conta dos alunos.

No segundo semestre de 2015 o professor de ASP é substituído. A partir das conversas iniciais sobre as experiências anteriores ele decidiu, a convite do docente de GP, aprofundar a experiência da interdisciplinaridade. O primeiro passo foi de fato transformar a proposta de intervenção em um trabalho único da disciplina, os dois professores iriam corrigir o mesmo trabalho integralmente e atribuiriam a mesma pontuação nas duas disciplinas.

No semestre seguinte, observou-se que para melhor andamento do projeto, as aulas deveriam ser planejadas pelos dois professores de forma que os conteúdos das duas disciplinas fossem apresentados em momento oportuno e de forma casada. Também se criou a figura dos "consultores", uma dupla de alunos que seria responsável por auxiliar a execução do projeto de outros colegas. Esta mudança visava a ampliação da troca entre os próprios alunos e do espaço para inovação. No mesmo sentido foram criados três momentos durante o semestre para que os alunos apresentassem o projeto em diferentes etapas do planejamento. Durante estas apresentações os professores, consultores e demais alunos apontavam sugestões para melhoria dos projetos. Ainda com intuito de propiciar um ambiente favorável a inovação e a experimentação, os professores definiram que as notas somente seriam 
lançadas após a entrega do trabalho final de forma que todos os erros durante o processo pudessem ser resolvidos até esta data, sem que o aluno recebesse nenhuma avaliação negativa.

Resumindo, durante um semestre letivo os alunos desenvolvem um Trabalho Interdisciplinar que consiste na formulação do marco lógico de um projeto de intervenção e de seu plano de gerenciamento. Para isso, os estudantes aplicam as ferramentas da MML e as áreas de conhecimento em gerenciamento de projetos. Os objetivos do trabalho são: aplicar os conceitos e ferramentas de elaboração, gestão e avaliação de projetos trabalhados nas disciplinas; estabelecer um problema de intervenção e realizar um diagnóstico descritivo sobre ele; desenvolver, a partir do diagnóstico, o modelo lógico de um projeto e o seu plano de gerenciamento; sistematizar o modelo lógico e o plano de gerenciamento num único documento de projeto; produzir um banner ou vídeo de curta duração sobre o projeto para exibição na Feira de Projetos e avaliação por professores e/ou gestores convidados.

O primeiro passo do trabalho é a escolha, por cada dupla de alunos, de um problema público de âmbito local, estadual ou nacional, discutido com os professores orientadores. Os estudantes desenvolvem os seguintes itens do projeto:

- Diagnóstico descritivo

- $\quad$ Árvore de Problemas

- $\quad$ Árvore de Objetivos

- $\quad$ Análise de Alternativas

- Matriz do Marco Lógico

- Declaração de Escopo e EAP

- Cronograma

- Orçamento

- Matriz de Responsabilidades

- Matriz de Planejamento de Riscos

- Matriz de Indicadores (qualidade)

- Matriz de Planejamento de Aquisições

- Matriz de Comunicação

- Plano de Avaliação

A etapa final do trabalho é a exposição das iniciativas, em formato banner ou vídeo, em Sessões Temáticas específicas, organizadas conforme os temas. Nelas, cada dupla de alunos, apresenta o projeto para uma banca, composta por um professor convidado e um servidor em exercício, que assistem o vídeo e fazem perguntas pertinentes ao trabalho. Os critérios de avaliação da exposição dos projetos são: (i) aplicação das metodologias de elaboração, gerenciamento e avaliação de projetos aprendidas; (ii) consistência da ideia do projeto; (iii) qualidade do banner/vídeo e discussão do projeto nas sessões temáticas.

A Feira de Projetos iniciou no segundo semestre de 2015 e já foram realizadas cinco edições. Ao todo 89 projetos foram elaborados, distribuídos em diferentes temática s, conforme tabela 1 abaixo:

Tabela 1 - Distribuição das temáticas dos projetos elaborados, Feira de Projetos, 2015-2017. 
Temáticas $\quad \mathbf{n}^{0}$ de projetos $\%$ do total

\begin{tabular}{|c|c|c|}
\hline Saúde & 13 & 14,61 \\
\hline Educação & 12 & 13,48 \\
\hline Segurança Pública & 10 & 11,24 \\
\hline Direitos humanos $^{1}$ & 8 & 8,99 \\
\hline Turismo e Cultura & 7 & 7,87 \\
\hline Assistência Social & 6 & 6,74 \\
\hline Trabalho & 6 & 6,74 \\
\hline Meio Ambiente & 6 & 6,74 \\
\hline Infraestrutura urbana & 5 & 5,62 \\
\hline Economia & 4 & 4,49 \\
\hline Habitação & 3 & 3,37 \\
\hline Mobilidade urbana & 3 & 3,37 \\
\hline Participação & 2 & 3,37 \\
\hline Gestão & 2 & 2,25 \\
\hline Regularização Fundiária & 1 & 1,12 \\
\hline Total & 89 & 100,0 \\
\hline
\end{tabular}

Fonte: Arquivo pessoal.

Nota: os projetos classificados na área de direitos humanos tratam sobre a população jovem, pessoas com deficiência, público LGBT, população carcerária.

Observa-se que pouco mais $1 / 3$ dos projetos realizados até o momento tratam de problemas de três áreas de saúde $(14,61 \%)$, educação $(13,48 \%)$ e segurança pública $(11,24 \%)$. $\mathrm{Na}$ saúde, destacam-se questões como "incidência de infecções sexualmente transmissíveis", "infraestrutura precária de equipamentos públicos de saúde" e "judicialização". Na educação, os problemas mais comuns são "evasão escolar", "baixa qualidade da educação", "violência nas escolas". Na área de segurança, são mais recorrentes projetos sobre "dificuldades do sistema penitenciário", "índices de roubo e furto", "uso e tráfico de drogas". Outros 1/3 dos projetos, que estão entre os $8 \%$ e $6 \%$ do total, propõem soluções para problemas dos setores de direitos humanos, turismo e cultura, assistência social, trabalho e meio ambiente.

\section{À guisa de conclusão: o que o caso ensina}

Ainda que de maneira incipiente pode-se observar que a Administração Pública por sua própria natureza é um campo fértil para o desenvolvimento da interdisciplinaridade. Não é possível estudá-la e ensiná-la distante de uma perspectiva interdisciplinar, mas sem se esquecer de que seu fundamento básico se ancora no contexto de um discurso eminentemente político.

A realidade extremamente complexa em que as instituições públicas atuam exige de seus praticantes a capacidade de articular teorias a partir de uma situação problema específica. $\mathrm{O}$ caso aqui discutido visa, em alguma medida, simular, ainda que no ambiente relativamente controlado da sala de aula, as vicissitudes da prática administrativa no setor público. Neste sentido, durante as discussões para elaboração do projeto de intervenção, os alunos refletem sobre questões como: quanto tempo deverá durar esta licitação?; qual a estimativa de custo para o desenvolvimento deste produto?; como buscar este conhecimento ou habilidade específica que não estão disponível na equipe de projeto identificada?; e seria oportuno a implementação deste projeto? Estes exemplos, embora corriqueiros, acionam 
conceitos, métodos e saberes desenvolvidos em diversas outras disciplinas do curso como: Administração Financeira e Orçamentária, Logística, Direito Administrativo, Gestão Pública, Teoria Política, entre outras. Deste modo, pode-se dizer que a realização da Feira de Projetos no sexto período favorece a busca deste ferramental dentro dos conhecimentos já acumulados pelos estudantes ao longo de sua formação.

Além disso, a apresentação dos projetos para gestores públicos em efetivo exercício é um momento de aprendizado substantivo, pois possibilita a discussão dessas questões fora da lógica utilitária do "professor corrigindo o trabalho", incentivando que os alunos argumentem as decisões tomadas no âmbito do projeto e desenvolvam habilidades de negociação e capacidade de exposição oral.

Do ponto de vista dos professores, observou-se que a experiência da interdisciplinaridade exige uma grande abertura para o outro, o que é conduta rara em muitas instituições de ensino, além de não fazer parte do processo de formação da maioria dos discentes. Pelo contrário, o que se observa nas instituições de ensino é que quase nunca dois professores dividem a mesma sala de aula, ou até mesmo dialogam para buscar soluções de ensino conjuntas. No espaço de trocas desenvolvido no âmbito da Feira, é necessário que os professores, em grande medida, abram mão da sua prerrogativa de autoridade, primeiro porque esta autoridade já começa compartilhada, segundo porque considerando a diversidade dos projetos, não é razoável esperar que algum professor seja capaz de conhecer profundamente a amplitude de temas abordados.

Do ponto de vista dos alunos, a abordagem adotada estabelece um grande desafio do rompimento com a lógica da divisão tradicionalmente disciplinar dos trabalhos acadêmicos. A organização de duplas, a complexidade do trabalho, assim como a interação entre as suas diversas partes favorecem o envolvimento dos alunos em todas as etapas. Além disto, a "consultoria" incentiva a troca entre eles, em espaços que ultrapassam a sala de aula.

Numa abordagem mais instrumental, observou-se ainda, indo além das questões de interdisciplinaridade, o desafio de combinar as questões teóricas e metodológicas com uma abordagem mais prática. As fases de delimitação do problema, assim como a definição do escopo são normalmente etapas críticas para a elaboração dos projetos de intervenção, sobretudo porque os problemas normalmente são muito complexos, mas as possibilidades de intervenção são bastante específicas, uma vez que estão sujeitas a restrições de tempo, escopo e custo. No mesmo sentido, as atividades que exigem mediação entre as prescrições bibliográficas e as práticas adotadas no setor público, como por exemplo: estimar a duração das atividades e especificar indicadores e metas, também apresentam dificuldades adicionais aos alunos que em alguma medida precisarão flexibilizar os conteúdos aprendidos em razão de dados obtidos no contexto de atuação do projeto.

Os principais potenciais da Feira de Projetos são propiciar que os estudantes articulem teoria e prática e que conheçam e experimentem as variáveis envolvidas no processo de tomada de decisão e de planejamento em políticas públicas. O caso ensina, portanto, ser possível aproximar a formação superior de administradores públicos com a prática profissional sem nem mesmo sair da sala de aula.

\section{Referências Bibliográficas}

Coelho, F. S. (2008). A problemática atual do ensino de graduação em administração pública no Brasil. Cadernos EBAPE.BR, Rio de Janeiro, número especial, p. 1- 21, ago. Disponível em: $<$ http://bibliotecadigital.fgv.br/ojs/index.php/cadernosebape/article/view/5435/4169>. Acesso em: 05 mar. 2019.

Fazenda, I. C. A. (2003). Interdisciplinaridade: história, teoria e pesquisa. 11. ed. Campinas: Papirus, $143 \mathrm{p}$.

Jannuzzi, P. M. (2005). Indicadores para diagnóstico, monitoramento e avaliação de programas sociais no Brasil. Revista do Serviço Público. Brasília, ano 56, n. 2, pp. 137-160, abr-jun. Disponível em: <https://revista.enap.gov.br/index.php/RSP/article/view/222/227>. Acesso em: 05 mar. 2018.

Jannuzzi, P. M. (2011). Avaliação de Programas Sociais no Brasil: repensando práticas e metodologias 
das pesquisas avaliativas. Planejamento e Políticas Públicas, Brasília. n. 36. jan-jul. Disponível em: $<$ http://www.ipea.gov.br/ppp/index.php/PPP/article/viewFile/228/212>. Acesso em: 05 mar. 2018.

Japiassu, H. (1976). Interdisciplinaridade e patologia do saber. Rio de Janeiro: Imago.

Mokate, K. (2002). Convirtiendo el 'monstruo' en aliado: la evaluación como herramienta de la gerencia social. Revista do Serviço Público. Brasília, ano 53, n. 1, p. 89-134, jan.-mar. Disponível em: $<$ http://repositorio.enap.gov.br/bitstream/1/1964/1/2002\%20Vol.53\%2cn.1\%20Mokate.pdf>

Oliveira, F. B.; Sant'anna, A.S.; Diniz, D. M. (2011). O ensino superior de administração pública: perspectiva histórica, características e possibilidades. In: ENCONTRO NACIONAL DE ENSINO E PESQUISA EM ADMINISTRAÇÃO E CONTABILIDADE, 3., 2011, João Pessoa. Anais... João Pessoa: ANPAD.

Oliveira, L. M. S. R.; Moreira, M. B. (2017). Da disciplinaridade para a interdisciplinaridade: um caminho a ser percorrido pela academia. Revista de Educação do Vale do São Francisco - REVASF. Petrolina, v.7, n. 12, p. 06-20. Disponível em: $<$ http://www.periodicos.univasf.edu.br/index.php/revasf/article/view/1006/666>. Acesso em: 05 mar. 2018.

Ortegón, E.; Pacheco, J. F. \& Prieto, A. (2005). Metodología del marco lógico para la planificación, el seguimiento y la evaluación de proyectos y programas. CEPAL, p. 13-28. Disponível em: <https://repositorio.cepal.org/bitstream/handle/11362/5607/4/S057518_es.pdf >. Acesso em: 05 mar. 2018.

Pfeiffer, P. (2000). O quadro lógico: um método para planejar e gerenciar mudanças. Revista do Serviço Público, Brasília, ano 51, n. 1, p. 81-12, jan-mar. Disponível em: < https://revista.enap.gov.br/index.php/RSP/article/view/320/326>. Acesso em: 05 mar. 2018.

Pfeiffer, P. (2005). Gerenciamento de Projetos de Desenvolvimento. Rio de Janeiro: Brasport.

Pires, V. et. al. (2014) Dossiê - Campo de Públicas no Brasil: definição, movimento constitutivo e desafios atuais. Administração Pública e Gestão Social, n. 6(3), p.110-126, jul-set. Disponível em: <http://www.apgs.ufv.br/index.php/apgs/article/view/719/371\#.WqMLcWrwbIU>. Acesso em: 05 mar. 2018.

Silva, D. J. (2000). O paradigma transdisciplinar: uma perspectiva metodológica para pesquisa ambiental. In: PHILIPPI JR., Arlindo. et. al. (Eds.). Interdisciplinaridade emCiências Ambientais. São Paulo: Signus, 2000. Cap. 4, p. 71-94. Disponível em: $<$ http://www.dominiopublico.gov.br/download/texto/us000001.pdf $>$. Acesso em: 05 mar. 2018. eletrico/hidroeletricidade>. Recuperado em: 6 jan. 2015. 